\title{
Ultrastable embedded surface plasmon confocal interferometry
}

\author{
Suejit Pechprasarn ${ }^{1}$, Bei Zhang ${ }^{1}$, Darren Albutt ${ }^{1}$, Jing Zhang $^{1}$ and Michael Somekh ${ }^{1,2}$
}

As disease diagnosis becomes more sophisticated, there is a requirement to measure small numbers of molecules attached to, for instance, an antibody. This requires a sensor capable not only of high sensitivity but also the ability to make measurements over a highly localized region. In previous publications, we have shown how a modified confocal microscope allows one to make localized surface plasmon (SP) measurements on a scale far smaller than the surface plasmon propagation distance. The present implementation presents a new ultrastable interferometer system, which greatly improves the noise performance. Hitherto, we have used the central part of the back focal plane to form a reference beam with the reradiated surface plasmons. In the current system, we block the central part and use the spatial light modulator to deflect s-polarized light into the pinhole to form an interference signal with the surface plasmons, thus creating an ultrastable interferometer formed with two beams incident at very similar angles. We demonstrate the superior noise performance of the system in hostile environments and examine further adaptations of the system to further enhance noise performance.

Light: Science \& Applications (2014) 3, e187; doi:10.1038/Isa.2014.68; published online 4 July 2014

Keywords: biosensor; common path; confocal microscopy; interferometry; surface plasmon

\section{INTRODUCTION}

In many biological sensing experiments, a measure of the sensitivity of a label free sensor, such as a surface plasmon (SP) sensor, is given in terms of the minimum detectable change in refractive index units. This gives an approximate measure of smallest detectable coverage (in mass per unit area) of a layer of analyte deposited on the sample; it does not account for the lateral extent over which the deposited analyte extends. It is now becoming apparent that many molecules such as cytokines are extremely powerful indicators of inflammatory response. Measurement of cytokine response can thus provide a powerful early diagnostic tool as well as having considerable potential in prognosis. Unfortunately, even though the relative change in cytokine concentration is large, the absolute concentration of cytokines is small compared to other species (by typically 6-8 orders of magnitude compared to commonly occurring plasma proteins). ${ }^{1}$ A sensor with poor lateral resolution requires a large number of analyte molecules compared to a sensor with similar sensitivity in terms of change in local refractive index where the measurement is highly localized. For this reason, there is a strong imperative to develop sensors with both improved sensitivity and lateral resolution. For instance, the use of heterodyne interferometry ${ }^{2,3}$ provides one approach to achieve this aim. Similarly, Yuan's group ${ }^{4}$ has developed several systems adapted to SP microscopic measurements. More recently, we have developed variants on confocal microscopy that perform in a similar manner to heterodyne interferometry but with simpler more compact instrumentation. In this paper, we present a microscopic plasmonic sensor which processes the optical beam paths to form a tightly focused common path interferometer with excellent immunity to external noise sources such as microphonics. The results presented in this paper were obtained on model non-biological samples to illustrate the operating principles and performance of the new configuration.

The system is operated in a defocused condition to ensure that the SPs propagate a substantial distance across the sample; however, the principal region of interaction remains tightly focused as the SPs come to a focus on the optical axis. ${ }^{5}$ We have shown previously ${ }^{6}$ how a defocused confocal microscope acts as an interferometer in which two principal optical paths form the two beams of an interferometer, which are shown as the black rays $\mathrm{P} 1$ and $\mathrm{P} 2$ in Figure $1 \mathrm{a}$ and $1 \mathrm{~b}$. These paths consist of a beam illuminating the sample close to normal incidence and another which is converted to a SP which propagates along the sample surface reradiating continuously. ${ }^{7}$ The confocal pinhole ensures that only the reradiated light that appears to come from the focus contributes to the detected signal. This confocal arrangement therefore ensures that the path of the SPs is well defined by the optical configuration rather than their propagation length. We have shown in subsequent publications ${ }^{8,9}$ how a spatial light modulator conjugate with the back focal plane is effective in allowing for different processing methods that overcome mechanical scanning or allow the amplitude and phase of the SP to be extracted directly, thus providing an exceptionally robust means of processing the signal. For instance, the spatial light modulator (SLM) can be used to change the phase of P1 relative to $\mathrm{P} 2$ so that phase stepping may be performed which allows the phase associated with SP to be extracted directly.

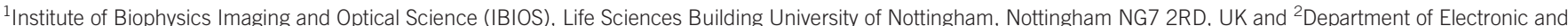
Information Engineering, Hong Kong Polytechnic University, Kowloon, Hong Kong, China

Correspondence: Professor M Somekh, Department of Electronic and Information Engineering, Hong Kong Polytechnic University, Kowloon, Hong Kong, China

E-mail: mike.somekh@nottingham.ac.uk or mike.somekh@polyu.edu.hk

Received 28 November 2013; revised 9 April 2014; accepted 12 April 2014 
Although the interference between $\mathrm{P} 1$ and $\mathrm{P} 2$ provides a relatively stable interferometer, the system sensitivity will, in many practical situations, be limited by microphonic noise, since both these beams hit the sample at different incident angles. Microphonic noise of amplitude $\Delta z$ introduces a phase shift given by:

$$
\Delta \phi=\frac{4 \pi n}{\lambda}\left(\cos \theta_{\mathrm{p} 1}-\cos \theta_{\mathrm{p} 2}\right) \Delta z
$$

where $\Delta \phi$ represents the phase noise, $n$ represents the refractive index of the coupling medium and $\lambda$ represents the wavelength of the incident light in vacuum, and $\theta_{\mathrm{p} 1}$ and $\theta_{\mathrm{p} 2}$ represent the incident angle associated with paths $\mathrm{P} 1$ and $\mathrm{P} 2$. From now on we denote $\theta_{\mathrm{p} 2}$ as $\theta_{\mathrm{p}}$ since it is associated with the angle at which SPs are excited.

In the implementation depicted in Figure 1a, where paths P1 and P2 interfere, $\theta_{\mathrm{p} 1}$ is very close to 0 , so that the first term in the brackets of Equation (1) can be replaced with 1 . The idea behind the present system is to produce an interferometer where the reference and signal beams are incident at angles which are very close to each other so that the terms in the brackets disappear or at least become very small. The system still retains sensitivity to changes in the wave number of the SP,

$$
k_{\mathrm{p}}=\frac{2 \pi n}{\lambda} \cos \theta_{\mathrm{p}}
$$

Clearly, as the ambient conditions change the value of $\theta_{\mathrm{p}}$ changes, so that the cancellation will become imperfect; however, in a normal binding experiment, where the change in $\theta_{\mathrm{p}}$ is small, the cancellation of microphonics will still be extremely good as quantified later in this paper.

In our previous publications, we have used linear input polarization in the back focal plane which gives a continuous variation between $p$ polarization and $s$-polarization as the azimuthal angle changes $6,8,9$ (Figure 2a). The use of radial polarization incident in the back focal plane has often been advocated in SP microscopy which means the light incident on the sample is $p$-polarized for all azimuthal angles, so that more energy is coupled in SPs and the focus is tight and circularly symmetrical. ${ }^{5}$ Our new interferometer, however, relies on linear polarization to generate interfering $p$ - and $s$-polarized beams hitting the sample at similar incident angles.

Examining Equation (1), we can see that an interferometer formed between a path generating a SP and one in which the incident light is primarily $s$-polarized will allow us to form an interferometer in which the sample and reference beams are incident on the sample at essentially the same angle of incidence. From Figure 1a, we can see that when the sample is defocused, the p-polarized light generates SPs, some of which appear to come from the focus to be collected by the confocal pinhole. In order to form our common path interferometer with the sample and reference beams incident at approximately the same angle, we remove path $\mathrm{P} 1$ and create a second path for the $s$-polarized light, which forms the reference arm of the interferometer. From Figure 1a, we see that the ray path corresponding to the $s$-polarized beam follows the path $\mathrm{P}^{\prime}$, so that on reflection, it does not propagate parallel to the optical axis, and will thus miss the pinhole. If an optical element is inserted into the return path that acts like a wedge (a linear phase gradient), it can be deflected parallel to the optical axis and will pass through the pinhole forming the reference beam of the interferometer. Alternatively, the optical element can be placed in the path of the incident beam, as shown in Figure 1b, so that its path follows $\mathrm{P} 1$ ' and hits the sample at point ' $\mathrm{b}$ ' so that on reflection it also appears to come from focus thus also forming an arm of the interferometer. ${ }^{6,8,9}$ In our experimental arrangement, the optical element (a spatial light modulator) was applied on the incident beam; thus corresponding to the scheme associated with Figure $1 \mathrm{~b}$.

Figure 2 shows a schematic of the pattern on the back focal plane used to effect the beam deflection. In positions where the light is primarily $p$-polarized, no linear phase gradient is applied as a portion of the reradiated SPs will appear to come from the focus. On the other hand, where light is predominantly s-polarized, it will interact with the wedge, thus ensuring that it follows path $\mathrm{P} 1$ ' of Figure $1 \mathrm{~b}$, thus returning to the pinhole. It is, of course, apparent that only along orthogonal directions is the incident light in a pure polarization state, for all other angles, there is continuous variation of the relative proportions of the two polarization states. This does not, however, produce any
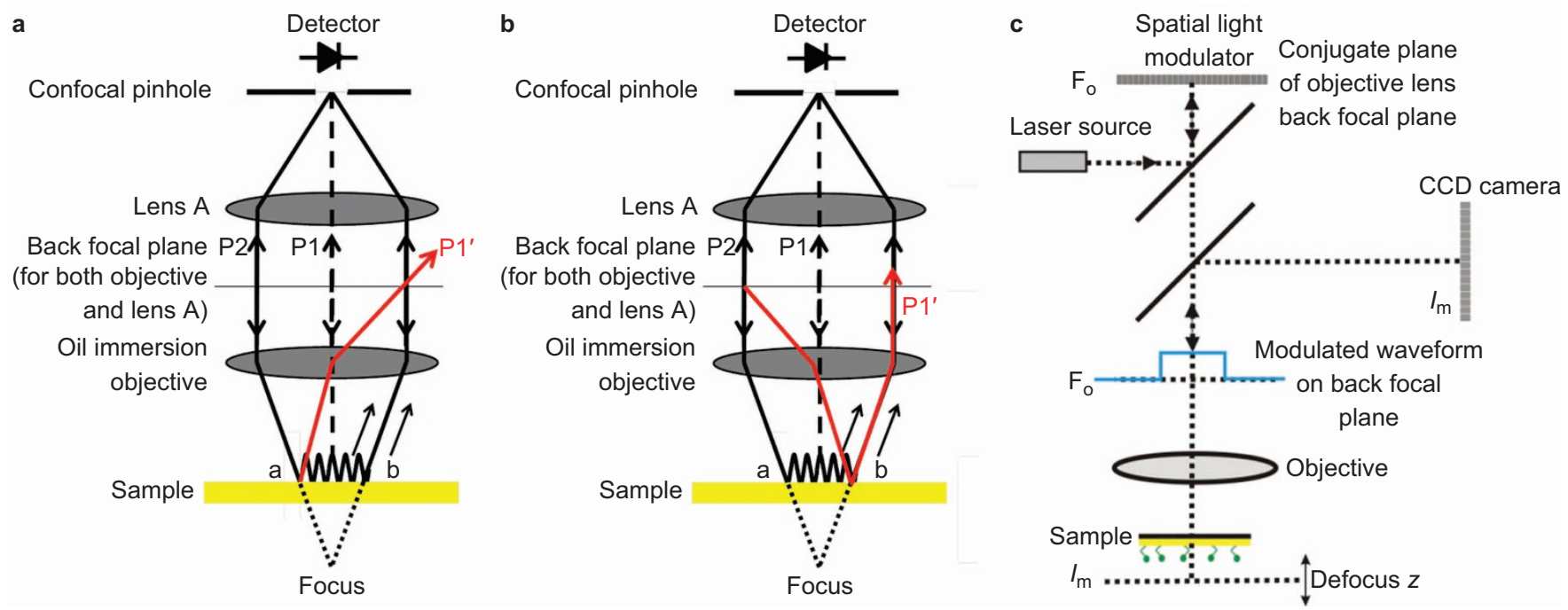

Figure 1 (a) Simplified schematic showing operation of a confocal microscope with SP excitation; the red lines indicate the direct reflection of incident s-polarization.

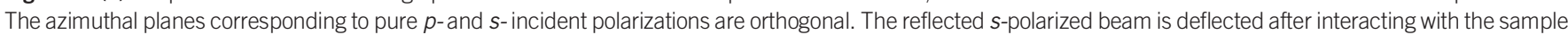

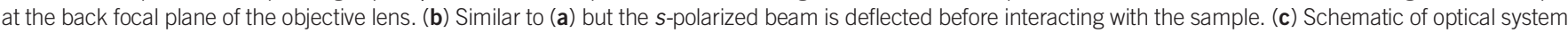

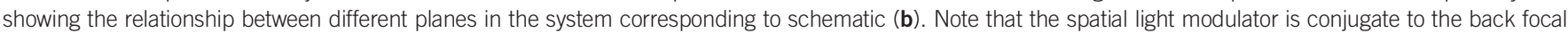
plane. The central part of the blue waveform indicates angles blocked by phase cancellation on the SLM. CCD, charge coupled device. 

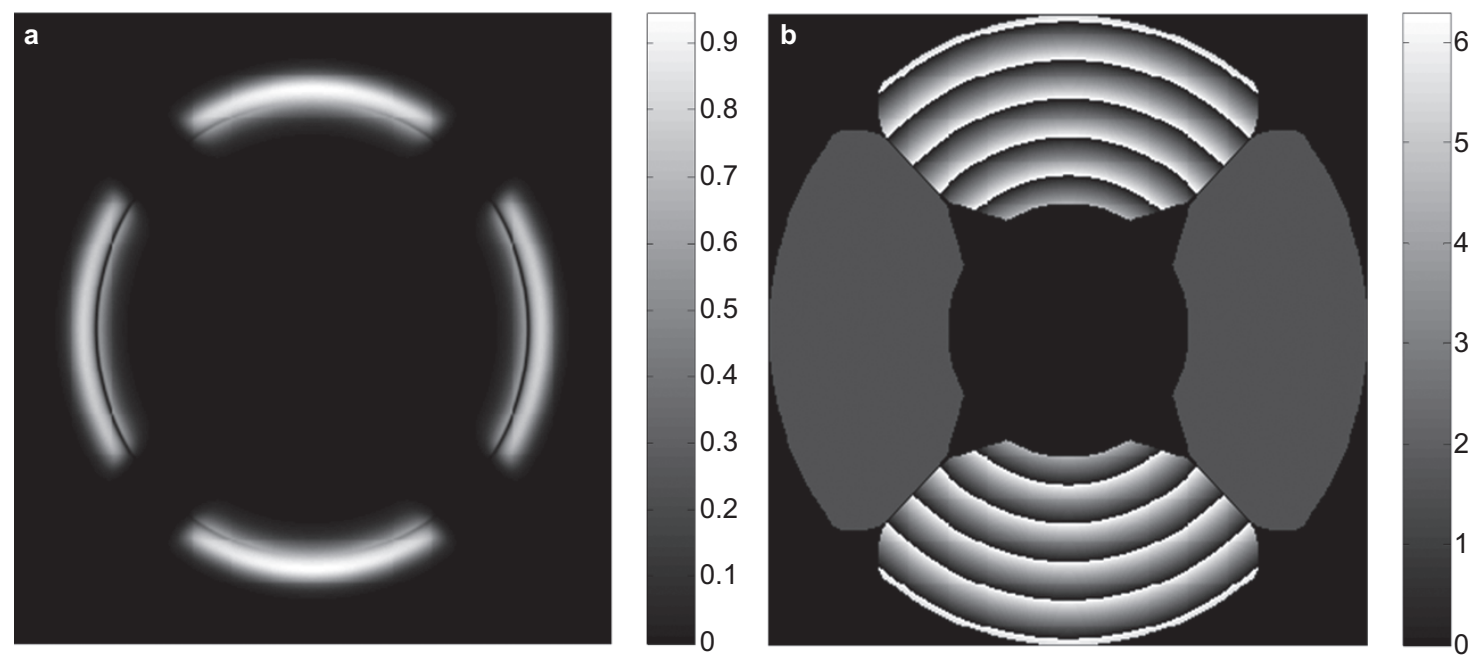

Figure 2 Amplitude and phase pupil function provided by phase spatial light modulator. (a) Amplitude pupil function and plasmonic dip position (dark band). (b) Phase gradient on s-polarization provides a wedge that bends the incident of $s$-polarized light so that its specular reflection passes through the pinhole, whereas there is no wedge in the path of the $p$-polarization; the wedge in the figure has an equivalent physical angle of $0.1^{\circ}$. Note that the $90^{\circ}$ phase steps were provided on the $p$-polarization region; (b) also shows $90^{\circ}$ phase modulation on the $p$-polarization. The horizontal direction corresponds to pure $p$-polarization and the vertical to pure $s$ polarization.

fundamental issues. As mention earlier, any incident s-polarization that does not interact with the wedge will miss the pinhole. Similarly, any p-polarized light interacting with the wedge will be deviated beyond the normal and miss the pinhole on the other side. This does, of course, entail some wastage of light.

We can now summarize the form of the pattern on the SLM. Firstly, only light close to the angle for excitation of SPs is allowed to pass, while other angles are blocked simply by setting adjacent pixels in antiphase as described in Refs. 8 and 9. The wedge is centered around the azimuthal angle corresponding to predominantly $s$-incident polarization, in the present work this angle corresponds to $\pm 45^{\circ}$. The wedge angle is controlled by the gradient of the phase shifts and the effect of varying this value is discussed later in the paper. In addition, we can phase shift one beam of the interferometer relative to the other by simply imposing a constant phase shift in the arc region corresponding to predominant $p$-polarization.

There are different ways of thinking about this approach, it may be thought of as generating an 'artificial' plasmon in that the spatial light modulator generates a continuously changing phase shift for the $s$ incident polarization that mimics the phase shift imposed by the sample when a SP is excited. ${ }^{10}$ Another way of thinking of the system is as a plasmonic analog of differential phase Nomarski microscopy; in this method, two adjacent regions on the sample are forced to interfere by passing through a polarization sensitive optical device. ${ }^{11}$ In the present technique, we similarly use the SLM to force two beams to interfere that would otherwise not interact. On a uniform sample, it is a matter of convenience whether the SLM is placed on the incident or reflected beam, although for a structured sample, the respective transfer functions differ. When the SLM acts before the sample, it is more natural to think of the approach from the 'artificial' plasmon viewpoint; since this is the implementation used in our work, we use the shorthand 'artificial' plasmon to describe our system.

It is important to note that confocal interferometry has been reported in the literature; ${ }^{12}$ however, this method uses a separate reference beam to interfere with the beam returning to the pinhole. In the work reported here and previously, ${ }^{8,9}$ the interference occurs between different parts of the illumination beam, so that one portion of the illumination beam acts as a reference. This approach facilitates our aim to obtain fine phase measurements between different paths within the same beam.

\section{MATERIALS AND METHODS}

The experimental set-up is essentially the same as described in Refs. 8 and 9 and is shown schematically in Figure 1c; the key difference in this paper is the manner in which the SLM (model no. BNS 512X512 phase; Boulder non-linear Systems Inc., Lafayette, Colorado, USA) is used to process the returning light by forming a wedge and performing the additional phase modulation as shown in Figure 2. Briefly, apart from the SLM, the key components in the system were the objective lens (1.45 numerical aperture (NA) oil immersion objective; Zeiss, Oberkochen, Germany), laser source (632.8 nm He-Ne laser, $10 \mathrm{~mW}$ ) and charge coupled device (CCD) camera (Sony Digital Interface XCL-S600C). It should be pointed out that all the experiments reported in this paper were obtained with air as the final medium; if measurements in aqueous media are required, it is necessary to use a higher NA objective such as the 1.49 NA oil lenses available from

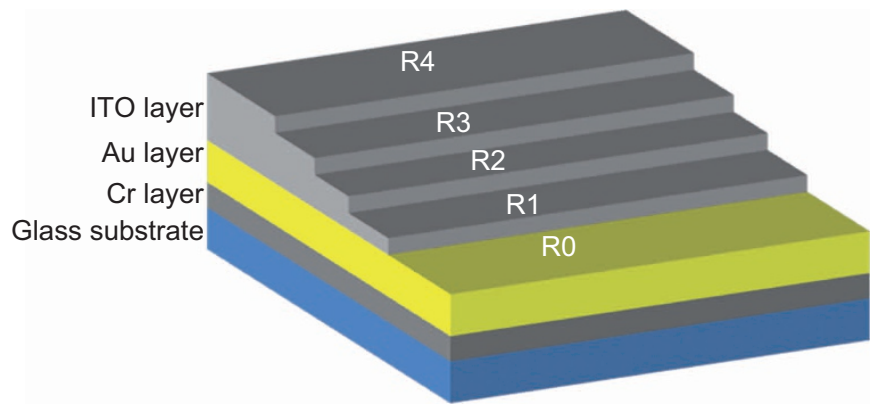

Figure 3 Schematic diagram of the sample, which consists of five different thicknesses of ITO layer including zero thickness deposited on uniform gold $50 \mathrm{~nm}$. The thickness of chromium $(\mathrm{Cr})$ adhesion layer was around $2 \mathrm{~nm}$. ITO, indium tin oxide. 
Nikon (Chiyoda, Tokyo, Japan). In order to evaluate the system, the sample shown schematically in Figure 3 was fabricated. Five different layer thicknesses (including zero) of indium tin oxide were deposited on the gold substrate used to support the SPs. In order to obtain an independent measure of the thickness of the layers, these were measured from the top surface using a spectroscopic ellipsometer (alpha-SE J. A.Woollam (Inc.), Lincoln, Nebraska, USA). We then measured the same sample with different configurations of the SP microscope. The first set of SP measurements used the approach described in Ref. 9 where a normally incident reference beam was used to interfere with the SP waves. Four $90^{\circ}$ phase steps were imposed on the reference beam so that a phase stepping algorithm could be used to extract the phase difference between the normally incident and the plasmonic contributions. This phase difference, $\phi$, can be plotted against the defocus, $z$. The mean gradient of this phase variation can be related to the angle of plasmon excitation, thus:

$$
\frac{\mathrm{d} \phi(z)}{\mathrm{d} z}=\frac{4 \pi}{\lambda}\left(\cos \theta_{\mathrm{r}}-\cos \theta_{\mathrm{p}}\right)
$$

where $\theta_{\mathrm{r}}$ is the mean incident angle of the reference beam $\left(\theta_{\mathrm{p} 1}\right.$ of Equation (1)), which is in this case zero.

\section{RESULTS AND DISCUSSION}

Figure 4 shows the measured values of $\phi(z)$ and from the gradients measured in the defocus region between -4000 and $-2000 \mathrm{~nm}$; we can obtain a value of $\theta_{\mathrm{p}}$ for each sample region by estimating the mean gradient of the curve and fitting to Equation (2). Note that negative values of defocus correspond to moving the sample closer to the objective relative to the focal point. If we assume the same refractive index as used to fit the ellipsometric data $(n=1.8583)$, we can recover a film thickness from the SP measurements from the Fresnel equations ${ }^{13}$ by calculating $\theta_{\mathrm{p}}$ for different layer thicknesses. It should be noted, of course, that unlike the ellipsometric measurements, the SP measurements are obtained from below the sample surface, that is, through the gold film. The thickness values obtained are tabulated in the second and third columns of Table 1 .

The agreement in the obtained thickness values between the ellipsometry measurements and the phase stepping measurements is good. Some of the discrepancy between the values arises from fact that the exact measurement position on the sample may differ.

We then used the 'artificial' plasmon to perform similar measurements on the stepped sample. Figure 5 shows the phase variation on

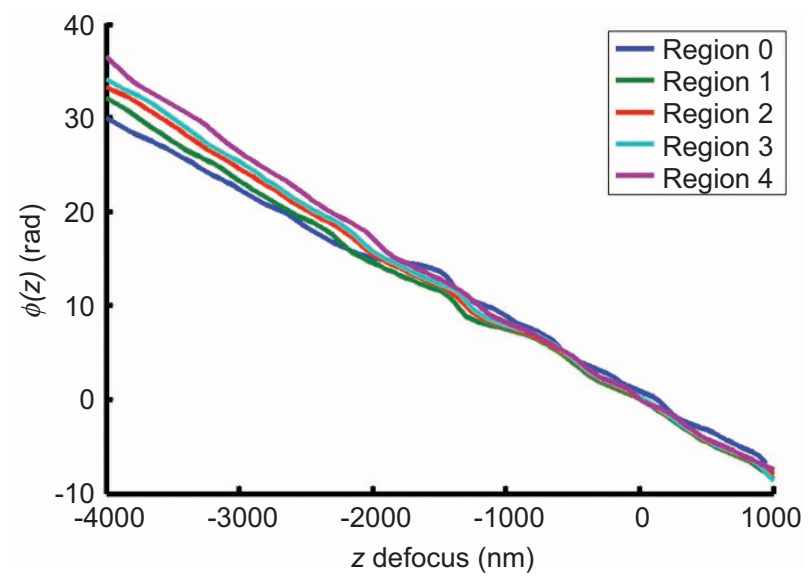

Figure 4 The measured values of $\phi(z)$ measured at the center of five different thicknesses of the ITO layer. ITO, indium tin oxide.
Table 1 Thickness values obtained by ellipsometric, phase stepping and artificial plasmon methods

\begin{tabular}{lccc}
\hline Region & Ellipsometry $(\mathrm{nm})$ & $\begin{array}{c}\text { Phase stepping } \\
(\mathrm{nm})\end{array}$ & $\begin{array}{c}\text { Artificial plasmon } \\
(\mathrm{nm})\end{array}$ \\
\hline R0 & 0.00 & 0.00 & 0.08 \\
R1 & 3.31 & 2.97 & 2.83 \\
R2 & 6.32 & 5.31 & 5.23 \\
R3 & 8.02 & 7.75 & 7.67 \\
R4 & 10.00 & 10.53 & 10.50 \\
\hline
\end{tabular}

region $\mathrm{R} 2$ of the coated sample for different wedge angles or phase gradients imposed in the back focal plane. The wedge was formed by a staircase pattern imposed on the SLM; the wedge angle was controlled by changing the gradient of the staircase. We see that from a defocus of $-1000 \mathrm{~nm}$ the gradient is much less than that obtained with the normally incident reference beam. We note, however, that there are two regions where the phase is stationary with defocus; we can also see that careful tuning of the wedge angle gives a flat region for a particular sample region. For regions R3 and R4, similar flat regions are obtained for larger wedge angles and for R0 and R1 for smaller wedge angles. The problem is that these regions of flat phase variation do not extend over large regions of defocus and methods to increase this range are discussed in the further developments section; nevertheless, this modest range of flat response was adequate to demonstrate the superior noise immunity of the present system. As we discussed in Ref. 9, the longer the range of defocus used to calculate the wave vector of the SP, the more accurate the measurement, on the other hand, reducing the maximum defocus improves the lateral resolution. ${ }^{5}$ We used the artificial plasmon method to measure the local gradient over a narrow range of defocus corresponding to the flat region from -2400 to $-2100 \mathrm{~nm}$. By phase stepping the region with predominant $p$-polarization, we obtained curves for the variation of $\phi(z)$ shown in Figure 6 for the five regions of the sample; we see that the R2 curve is approximately horizontal, whereas R0 and R1 have positive gradients and R3 and R4 have negative gradients as expected. We can use the measured gradients with Equation (2) with $\theta_{\mathrm{r}}$ equal to $\theta_{\mathrm{p}}$ for the R2 region. These values are shown in the final column of Table 1, which show excellent agreement with results involving a normally incident reference beam.

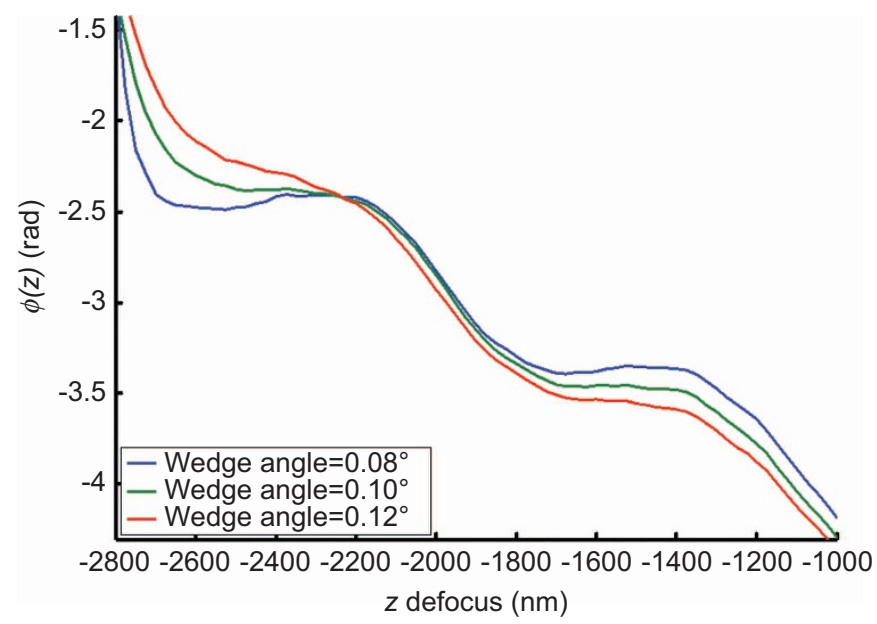

Figure 5 The phase variation on region R2 of the coated sample for different effective wedge angles. 


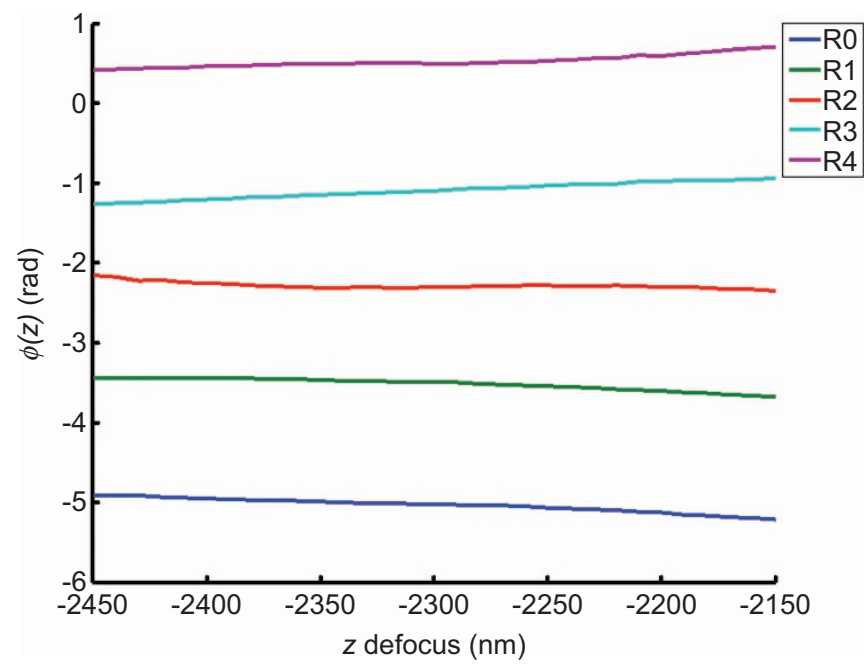

Figure 6 Variation of the phase output from the interferometer, $\phi(z)$, for the five regions of the sample, where the system was tuned so that the $\phi(z)$ slope of the region $\mathrm{R} 2$ was relatively flat.

We now consider the issue of noise immunity which we believe is the principal advantage of the new technique. The results above were all obtained with our optical table pumped up, to test the noise performance the legs of the optical table were deflated and a large bass speaker was placed on the table to induce large amounts of microphonic noise. The vibration was measured with a commercial Polytec, Waldbronn, Germany vibrometer sensor head (OFV 534) and controller (OFV 5000) illuminating the sample holder; when the table was pumped up and the speaker was off, the root mean square (rms) vibration was less than $10 \mathrm{~nm}$, while with the speaker on and the table deflated, the rms vibration was $270 \mathrm{~nm}$ with peak to peak excursions of approximately $760 \mathrm{~nm}$. The response waveform was approximately sinusoidal at $20 \mathrm{~Hz}$.

To get an estimate of the noise in thickness measurements, we measured the noise in the $\phi(z)$ curves from the deviation of the measured phase shifts from their best linear fitted results. We then used this noise variance to generate a new set of data with the same underlying statistics; this was then used to get another estimate of $\phi(z)$ from which a new estimate of thickness was obtained. This process was repeated $10^{6}$ times to get the noise statistics presented in Table 2.

From Table 2, we see a dramatic improvement in noise performance in the 'artificial' plasmon system. Several points emerge from this table:

- The results for $\mathrm{R} 2$, where the wedge angle is best matched to the expected value of $\theta_{p}$, give a noise standard deviation 54 times smaller compared to the $1000 \mathrm{~nm}$ scan and 35 times less compared to the $2000 \mathrm{~nm}$ scan. This improvement was achieved even though

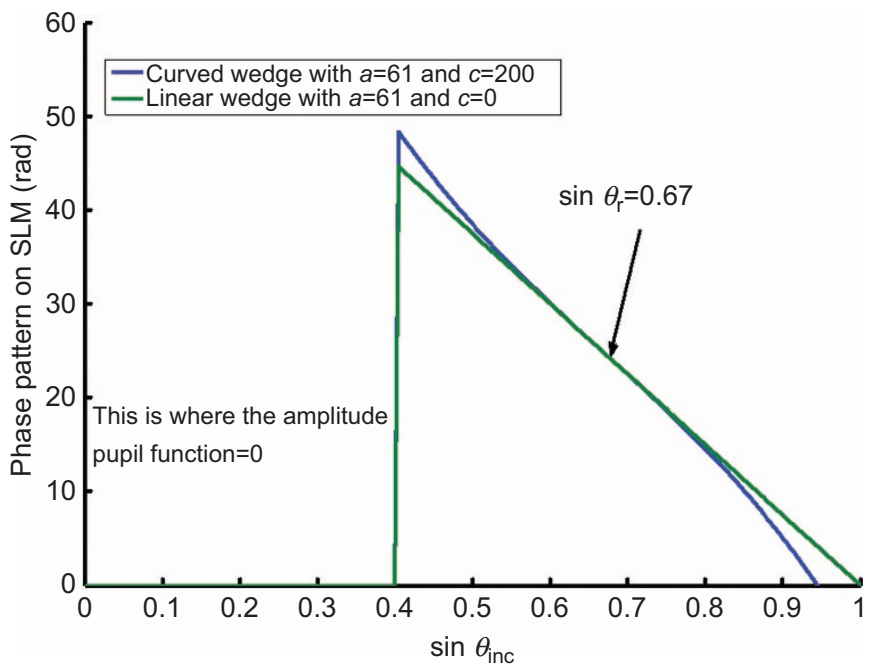

Figure 7 Linear wedge and curved wedge showing phase variation vs. sine of the incident angle determined by radial position in the back focal plane, where the curved wedge can be generated using third order polynomial equation with $a=61, c=200$ and $\sin \theta_{\mathrm{r}}=0.67$. Solid green for linear wedge and solid blue for curved wedge. Note that for the 1.49 NA objective and coupling oil with index 1.52 , the maximum value of $\sin \theta_{\mathrm{r}}$ is $0.98\left(\theta_{\mathrm{r}} \approx 78.5 \%\right.$. NA, numerical aperture; SLM, spatial light modulator.

the scan range for the artificial plasmon experiment was only $300 \mathrm{~nm}$. Extending the 'flat' artificial plasmon range will improve the signal to noise even more as demonstrated in Ref. 5 for the system with the normal reference beam. Approaches to extend the flat region are discussed below.

- The improvement in noise performance in other regions, while still good, was not as great as for region R2; this is to be expected at the wedge angle: in these cases, it is less well matched to $\theta_{\mathrm{p}}$. The noise in the normal incidence phase stepping system is approximately constant on each sample region which is a consequence of the fact that the microphonics are not cancelled.

In a typical biomedical experiment, the values of $\theta_{\mathrm{p}}$ vary by small amounts so the noise immunity provided by a single wedge angle is likely to apply to the whole measurement. If this were not the case, the use of the electronically programmable SLM means that it is easy to vary the wedge angle interactively as the measurement proceeds. The system as presented thus has the potential for optimal performance in terms of sensitivity, spatial resolution and dynamic range. We believe that these three key characteristics have not hitherto been achieved in a single system.

\section{Extending the flat slope defocus}

Up to this point, we have used a linear wedge in all the measurements which gives a measurement range with good phase cancellation of

Table 2 Standard deviation $\sqrt{\sigma^{2}}$ of thicknesses of the sample

\begin{tabular}{|c|c|c|c|}
\hline \multirow{2}{*}{ Sample region } & \multicolumn{3}{|c|}{ Standard deviation $\sqrt{\sigma^{2}}$ of thicknesses in $\mathrm{nm}$} \\
\hline & Phase stepping $z$ defocus distance $=2000 \mathrm{~nm}$ & Phase stepping $z$ defocus distance $=1000 \mathrm{~nm}$ & Artificial plasmon $z$ defocus distance $=300 \mathrm{~nm}$ \\
\hline RO & 0.1053 & 0.1761 & 0.0098 \\
\hline R1 & 0.1084 & 0.1688 & 0.0032 \\
\hline $\mathrm{R} 2$ & 0.1053 & 0.1621 & 0.0030 \\
\hline R3 & 0.1145 & 0.1823 & 0.0089 \\
\hline R4 & 0.1022 & 0.1582 & 0.0132 \\
\hline
\end{tabular}




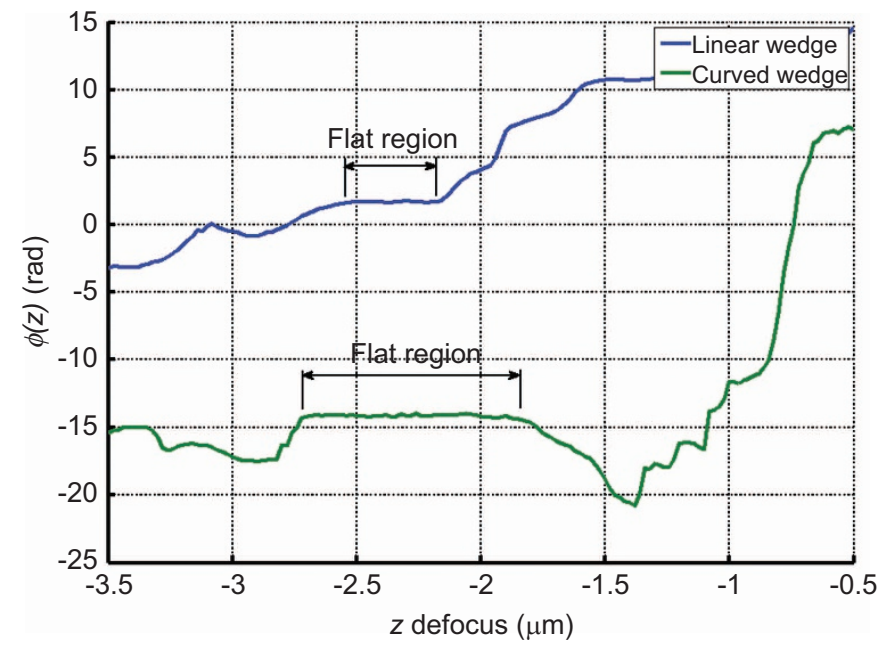

Figure $8 \phi(z)$ curves corresponding to the wedges in Figure 7. Solid blue for linear wedge and solid green for curved wedge obtained on a bare gold sample.

around $300 \mathrm{~nm}$. In this section, we will show that this flat region can be extended by using a 'curved' wedge that deviates from the perfect linear case, such as the one shown in Figure 7. This is represented by adding a simple cubic term to introduce some curvature as shown in Equation (3).

$$
\phi(\sin \theta)=a\left(\sin \theta-\sin \theta_{\mathrm{r}}\right)+c\left(\sin \theta-\sin \theta_{\mathrm{r}}\right)^{3}
$$

where $a$ is the gradient of the wedge and the $c$ is the parameter that is varied to get optimum region with flat phase response. The comparison between the linear wedge and the curved wedge is shown in Figure 8 , where we can see that the curved wedge can extend the flat region to around $1 \mu \mathrm{m}$. These results were obtained using a 1.49 NA oil immersion objective (Nikon) and laser source (690 nm laser).

\section{CONCLUSIONS}

This paper has presented a technique to overcome the microphonic phase error in a confocal SP microscope, where the reference beam is provided by direct reflection of $s$-polarization at an angle similar to the plasmonic angle. In order to force the s-polarised light into the pinhole, we provide a wedge that interacts with the s-polarised light, achieved conveniently with a phase SLM. This method can be combined with phase stepping to obtain a highly sensitive measure of the $\mathrm{SP}$ wave vector. We have shown that the present technique gives at least an order of magnitude better noise immunity than the phase stepping method published in Ref. 9, despite using the same optical set-up. We have also demonstrated that further optimization in terms of increased range of noise immunity can be achieved with relatively subtle, but simply implemented, changes to the patterns imposed on the SLM. We believe that this is a major step for making robust and sensitive plasmonic measurements over small local regions, thus ultimately reducing the minimum number of detectable molecules adhering to the surface.

\section{ACKNOWLEDGEMENTS}

We acknowledge Engineering and Physical Sciences Research Council for funding this work in the form of a platform grant entitled 'Strategies for optimal Biological Imaging', a University of Nottingham Hermes Innovation Fellowship and the China Scholarship Council for funding for BZ. We are grateful to Professor Aaron Ho (Chinese University of Hong Kong, Hong Kong) for valuable discussions.

1 Anderson NL, Anderson NG. The human plasma proteome: history, character, and diagnostic prospects. Mol Cell Proteom 2002; 1: 845-867.

2 Argoul F, Monier K, Roland T, Elezgaray J, Berguiga L. High resolution surface plasmon microscopy for cell imaging. Proc SPIE 2010; 7715: 771506.

3 Somekh MG, Liu SG, Velinov TS, See CW. High-resolution scanning surface-plasmon microscopy. Appl Opt 2000; 39: 6279-6287.

4 Zhang CL, Wang R, Wang YJ, Zhu SW, Min CJ et al. Phase-stepping technique for highly sensitive microscopic surface plasmon resonance biosensor. Appl Opt 2014; 53: 836-840.

5 Pechprasarn S, Somekh MG. Surface plasmon microscopy: resolution, sensitivity and crosstalk. J Microsc 2012; 246: 287-297.

6 Zhang B, Pechprasarn S, Zhang J, Somekh MG. Confocal surface plasmon microscopy with pupil function engineering. Opt Express 2012; 20: 7388-7397.

7 Lambeck AJ, Crijns AP, Leffers N, Sluiter WJ, ten Hoor KA et al. Serum cytokine profiling as a diagnostic and prognostic tool in ovarian cancer: a potential role for interleukin 7. Clin Cancer Res 2007; 13: 2385-2391.

8 Zhang B, Pechprasarn S, Somekh MG. Surface plasmon microscopic sensing with beam profile modulation. Opt Express 2012; 20: 28039-28048.

9 Zhang B, Pechprasarn S, Somekh MG. Quantitative plasmonic measurements using embedded phase stepping confocal interferometry. Opt Express 2013; 21: 1152311535.

10 Somekh MG. Surface plasmon and surface wave microscopy. In: Optical Imaging and Microscopy. Berlin/Heidelberg: Springer; 2007. pp347-399.

11 Allen RD, David GB, Nomarski G. The zeiss-Nomarski differential interference equipment for transmitted-light microscopy. Z Wissen Mikrosk Mikroskop Tech 1969; 69: 193-221.

12 Fischer DG, Ovryn B. Interfacial shape and contact-angle measurement of transparent samples with confocal interference microscopy. Opt Lett 2000; 25: 862.

13 Hecht E. Optics. Reading MA: Addison-Wesley Longman, Inc.; 2002.

\section{(c) (i) (2) This work is licensed under a Creative Commons Attribution-}

cc) party material in this article are included in the article's Creative Commons license, unless indicated otherwise in the credit line; if the material is not included under the Creative Commons license, users will need to obtain permission from the license holder to reproduce the material. To view a copy of this license, visit http://creativecommons.org/licenses/by-nc-sa/3.0/ 